\title{
An Infectious Disease Process within an Immune- Mediated Disease Process: Role of the Gastrointestinal Microbiota in Crohn's Disease
}

\author{
Gilles RG Monif* \\ Infectious Diseases Incorporated, Nebraska
}

Submission: May 06, 2017; Published: June 13, 2017

"Corresponding author: Gilles R. G. Monif, Infectious Diseases Incorporated, is 17121 Lakewood Drive Bellevue Nebraska 68123,

E-mail: gmonif@AOL.com,gmonif@AOL.com

\section{Mini Review}

The Hruska Postulate states that Crohn's disease is the consequence of immune-mediated destruction of gastrointestinal mucosa at the principal points of Mycobacterium avium subspecies paratuberculosis (MAP) attachment and antigen processing [1]. Focal destruction of mucosal integrity is the immune-mediated portion of Crohn's disease. MAP epithelial binding receptor sites line the entire small intestine [2]. MAP mucosa attachment is greatest in areas of significant fecal stasis. The mucosa's immense regenerative capacity of the lining epithelium renders quantitative limited and widely spaced MAP antigen challenges to be of transient significance. Increasing inoculum loads and more closely spaced antigen challenges eventually overwhelm the ability of the mucosa to heal.

In Crohn's disease, gastrointestinal symptomology is a function of gastrointestinal mucosal integrity. Mucosal healing correlates with clinical remission, whereas significant denudement is associated with the presence of Crohn's disease's clinical spectrum of signs and symptoms. Once mucosal integrity is significantly breached, the intra-luminal gastrointestinal microbiota invades lamina propria and submucosa. What happens is that an immune mediated disease process creates a secondary infections disease process that together produce the pathogenic spectrum of disease.

Bacterial groupings that exist within the contained physical space exist in a dynamic, but highly regulated relationship to each other [3-6]. Changes in the intraluminal environment can alter the inter-relationships [7]. In cattle, grain feeding, as opposed to grass consumption, allows members of the Enterobacteriace (e.g. Escherichia coli) to attain greater prevalence. Loss of the anatomical barrier separating the gastrointestinal microbiota from the underlying tissues, coupled with inflammation-induced reduction of the local oxygen-reduction potential, alters the microbiological hen-peck order among microbes, and initiates a confrontation between invasive organisms and embedded mucosal immunity. Once polymicrobial invasion has occurred, the dominant bacteria further alter the local microbiological environment and readdress the microbial hen-peck order through a process known as the anaerobic progression [8-10].

The anaerobic progression is best exemplified by what happens in women who contract acute infection by Neisseria gonorrheae. The inflammatory response resulting from gonococcalendocervicitis so alters the microbiological environment as to allow recruitment of bacteria with the microbial flora of the female genital tract $[11,12]$. Their selective replication further alters the microbiological environment such that the more facultative bacteria undergo elimination and are replaced by more obligatory anaerobes $[13,14]$. The potential for the anaerobic progress to cause endometritis, salpinitis and peritonitis is why antibiotic therapy for men and women with acute gonococcal infection is so divergent [15]. With access to the resident vaginal microbiological flora, monoetiological disease is transiently transformed into polymicrobial disease. In Crohn's disease, immune destruction of the gastrointestinal mucosa and the resultant inflammation functions to set in motion and give access to the anaerobic progression.

If epithelial reconstitution is achieved relatively early, host immunity usually re-establishes microbial control. Eighty percent of the body's immune system resides in the gastrointestinal tract. It is a dynamic interplay of checks and balances. Intestinal immune regulation is achieved through opposing arms of the immune system. By their penetration, microbes initiate a pro-inflammatory T-cell response with the development of T-helper-1 (TH1, TH2 and/or TH17) cells. These pro-inflammatory cells become counterbalanced by 
the elaboration of regulatory inducible T-cells (Tregs) that have variable Foxhead Box transcription factor (Foxp3f). Treg differentiation results in production of anti-inflammatory cytokines. In experimental colitis models, suppression of TH1 and TH17 cell responses prevent the inflammatory response from developing. Steroids and biologics that can induce remissions, directly or indirectly, target effecter T-cells or neutralize selected cytotoxic cytokines elaborated. In the absence of bacteria with direct or indirect enhanced virulence, epithelial reconstitution of the anatomical barrier gives leverage to mucosal immunity to terminate microbial invasion. If access to the bacterial flora of the gastrointestinal tract is cut off by mucosal healing rapidly, gut immunity has the potential to terminate the ongoing infectious disease process.

If microbial invasion is not arrested in a timely framework, residual bacterial induced damage affects clinical symptomology. Damage mediated primarily by the Enterobacteriace results in fibrosis with impaired bowel motility and ultimately stricture formation. Bowel perforation, loop-to-loop intestinal fistula, and abscesses are products of obligatory bacterial and not MAP. One has only to look at the gastrointestinal pathology caused by MAP in cattle (Johne's disease) to understand the significant contribution to morbidity and complication imposed by inadequately addressing the invading gastrointestinal microbiota. MAP does not cause loop-to-loop intestinal fistulae, fistula, bowel perforation or strictures.

Based upon the Hruska Postulate, the therapeutic objectives in Crohn's disease are:

I. Re-establish mucosal integrity,

II. Eradication of the induced polymicrobial infection, and

III. Destruction of the MAP template that sustains the dysfunctional, pro-inflammatory cytokine cascade against MAP's antigenic array.

Of the three, eradication of polymicrobial super infection is the least served therapeutically.

\section{References}

1. Monif GRG (2015) The Hrusha postulate of Crohn's disease. Med Hypothesis 85(6): 878-881.
2. Schleig PM, Buergelt CD, Davis JK, Williams E, Monif GR (2005) Attachment of Mycobacterium avium subspecies paratuberculosis to bovine intestinal organ cultures: method development and strain differences. Vet Microbiol 108(3-4): 271-279.

3. Chaisilwattana P, Fagnant JE, Monif GRG (1991) Bacterial interference by group B streptococci with aerobic and anaerobic genital tract streptococci. Thai J Obstet Gynecol 3: 45-52.

4. Chaisilwattana P, Monif GRG (1995) In vitro ability of the group B streptococci to inhibit gram-positive and gram-negative constituents of the female genital tract bacterial flora. Infect Dis Obstet Gynecol 3: 91-97.

5. Larsen B, Monif GRG (2001) Understanding the bacterial flora of the female genital tract. Clin Infect Dis 32: 69e-77e.

6. Carson HJ, Lapoint PG, Monif GRG (1997) Inter-relationships with the bacterial flora of the female genital tract Infect. Dis Obstet Gynecol 5(4): 305-309.

7. Holdeman LV, Good IJ, Moore WE (1976) Human fecal flora: variation in bacterial composition within individuals and possible effect of emotional stress. Appl Environ Microbiol 31(3): 359-375.

8. Gorbach SL, Mayhew JW, Bartlett JG, Thadepalli H, Onderdonk AB (1974) Rapid diagnosis of anaerobic infections by direct gas-liquid chromatography of clinical speciments. J Clin Invest 57(2): 478-484.

9. Gorbach SL, Bartlett JG (1974) Anaerobic infections. N Engl J Med 290: 1280-1294.

10. Gorbach Sl (1979) Clinical aspects of anaerobic infections. J Infect 1: 25s-37s.

11. Chow AW, Maikasian KL, Marshall JR, Guze LB (1975) The bacteriology of acute pelvic inflammatory disease-value of cul-de-sac cultures and relative importance of gonococci and other aerobic or anaerobic bacteria. Am J Obstet Gynecol 122: 876-881.

12. Monif GRG, Welkos SL, Baer H (1977) The bacteriologic spectrum of isolates obtained from the cul-de-sac of patients with endometritissalpingitis-peritonitis. Excerta Medical 3: 26-29.

13. Monif GR, Welkos SL, Baer H (1977) Clinical response of patients with gonococcal endocervicitis and endometritis-salpingitis-peritonitis to doxycycline. Amer J Obstet Gynecol 129(6): 614-622.

14. Monif GR (1980) Significance of polymicrobial superinfection in the therapy of gonococcal endometritis-salpingitis-peritonitis. Obstet Gynecol 55(5 Suppl): 154s-161s.

15. Monif GRG (2001) Pelvic inflammatory disease redefined. Infect Med 18: $532-533$
This work is licensed under Creative Commons Attribution 4.0 License

DOI: 10.19080/ARGH.2017.05.555675

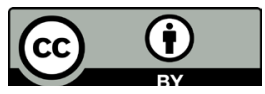

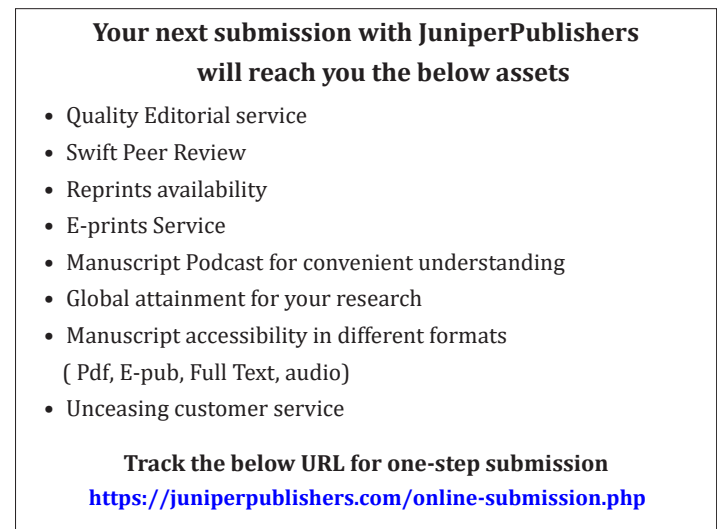

